\title{
APLICACIÓN DE UNA TÉCNICA GEOFÍSICA (GEORRADAR) PARA EL ESTUDIO DE BIOMASA SUBTERRÁNEA EN ECOSISTEMAS COSTEROS
}

\author{
APPLICATION OF TECHNICAL GEOPHYSICAL (GEORRADAR) FOR THE \\ STUDY OF UNDERGROUND BIOMASS IN COASTAL ECOSYSTEMS
}

Luís Fernando, Romero Castellano

Ing. Ambiental, Ph.D, Departamento de ingeniería civil y ambiental, Universidad de Pamplona (Colombia).E-mail: luisromero@unipamplona.edu.co

Pedro Javier, Jáuregui Eslava

Ing. Caminos Canales y Puertos, Ph.D., Departamento de ciencias de la tierra y del medio ambiente, Universidad de Alicante (España). E-mail: pedro.jauregui@ua.es

Jacipt Alexander, Ramón Valencia

Ing. Químico, Ph.D., Departamento de ingeniería civil y ambiental, Universidad de Pamplona (Colombia).

Fecha de recepción: 7 abril de 2008.

Fecha de aprobación: 16 de junio de 2008.

\section{RESUMEN}

Comúnmente desarrollar investigaciones del sistema radicular por métodos tradicionales implica utilizar técnicas destructivas y bastante laboriosas, estudios que en muchos casos ocasionan impactos ambientales considerables en cualquier ecosistema y más en sistemas frágiles como los dunares. En este estudio se presenta y desarrolla una metodología para el estudio de las raíces de dos especies arbóreas Pinus halepensis Miller y Pinus pinea L, técnica compatible con el medio dunar. Los resultados obtenidos en esta investigación usando este tipo de tecnologías no invasivas-no destructivas, aportarán información sobre la distribución y profundidad que alcanzan las raíces en este medio natural. Incluso esta información puede servir a futuro para estimar la biomasa subterránea, permitiendo ahorrar una gran cantidad de tiempo, esfuerzo e incluso dinero.

Palabras clave: Georradar, biomasa, dunas, tecnologías no invasivas, radargrama. 


\section{ABSTRACT}

Traditional develop investigations of the root system by means using traditional methods and destructive techniques quite laborious, studies that often cause significant environmental impacts in any ecosystem and more fragile as the dune systems. In our present study and develop a methodology to study the roots of two tree species Pinus halipensis Miller and Pinus pinea $L$, technical compatible with the middle dunes. The results obtained in this investigation using this type of technology non invasive, provide information on the distribution and depth that reaches the roots in this environment. Even this information can serve to estimate future below-ground biomass, allowing save a lot of time, effort and even money.

Key words: G.P.R, roots, technology non invasive, dune, biomass.

\section{INTRODUCCIÓN}

El georradar es un sistema basado en la emisión y recepción de ondas electromagnéticas en el suelo y capaz de producir una imagen (radargrama) de los elementos que hay bajo el mismo, es una técnica que se utiliza en investigaciones poco profundas. El empleo del georradar o G.P.R se ha popularizado a partir de los años 70 cuando aparecieron publicados los primeros trabajos en minería y geología [2], [16]. Sobre los años 80 y 90 esta técnica adquirió mayor relevancia en estudios medioambientales y de arqueología [5], [7], [8], [14], [18]. A partir del año 1999 se inician las aplicaciones de esta técnica en el campo de la ecología, investigadores como Hruska et al. [9], hacen un análisis de las raíces de la especie Quercus petraea (Mattuschka) Liebl, y Butnor et al [4], indagó sobre el sistema radicular de pino (Pinus taeda L.).

Los ecosistemas dunares son ambientes más frágiles, con mayor posibilidad de sufrir una interacción que derive en la alteración de su dinámica litoral, en los que cobran notable protagonismo las acciones humanas, como modificadores de su evolución [11]. Ante las numerosas alteraciones sufridas, estos espacios han tenido una importancia progresiva en los últimos años en cuanto a iniciativas por resguardar sus valores [13]. Una de las características del sistema dunar son sus suelos, pobres en nutrientes y baja capacidad de retención de agua [3], [10], [12], [17], esta variable está influenciada por su particular granulometría o el contenido de materia orgánica [6], [15], la movilidad de la arena [17], temperaturas variables [19] y la edad de las dunas [17].

El objetivo de la investigación es evaluar la utilidad del georradar (G.P.R) en el estudio del sistema radicular de las dos especies arbóreas dominantes en el sistema dunar. 


\section{MATERIAL Y METODOS.}

El estudio se realizó en el pinar de Guardamar de la Segura (38 06.128' N y $0^{\circ} 38.965^{\prime} \mathrm{W}$ ) (España), a $45 \mathrm{Km}$ al SE de la ciudad de Alicante, en el municipio del mismo nombre. El sistema dunar estudiado ocupa a lo largo de la costa del Mediterráneo, una faja de unos $13 \mathrm{Km}$. y un ancho entre 500 y algo más de 1000 metros, con alturas de hasta $15 \mathrm{~m}$. El área total del ecosistema dunar son unas 900 hectáreas aproximadamente [1] figura 1. En la actualidad el ecosistema presenta un amplio dominio por las especies de estrato arbóreo siendo sus principales representantes Pinus halepensis Miller y Pinus pinea L fruto de la restauración. Además existe un claro predominio de plantas herbáceas como Teucrium capitatum, Thymus vulgaris y Fagonia cretica. En los claros además de los géneros ya mencionados se pueden observar Asparagus acutifolius, Asphodelus fistolosus, Sideritis leucantha, Hordeum murimum y Brachypodium retusum.

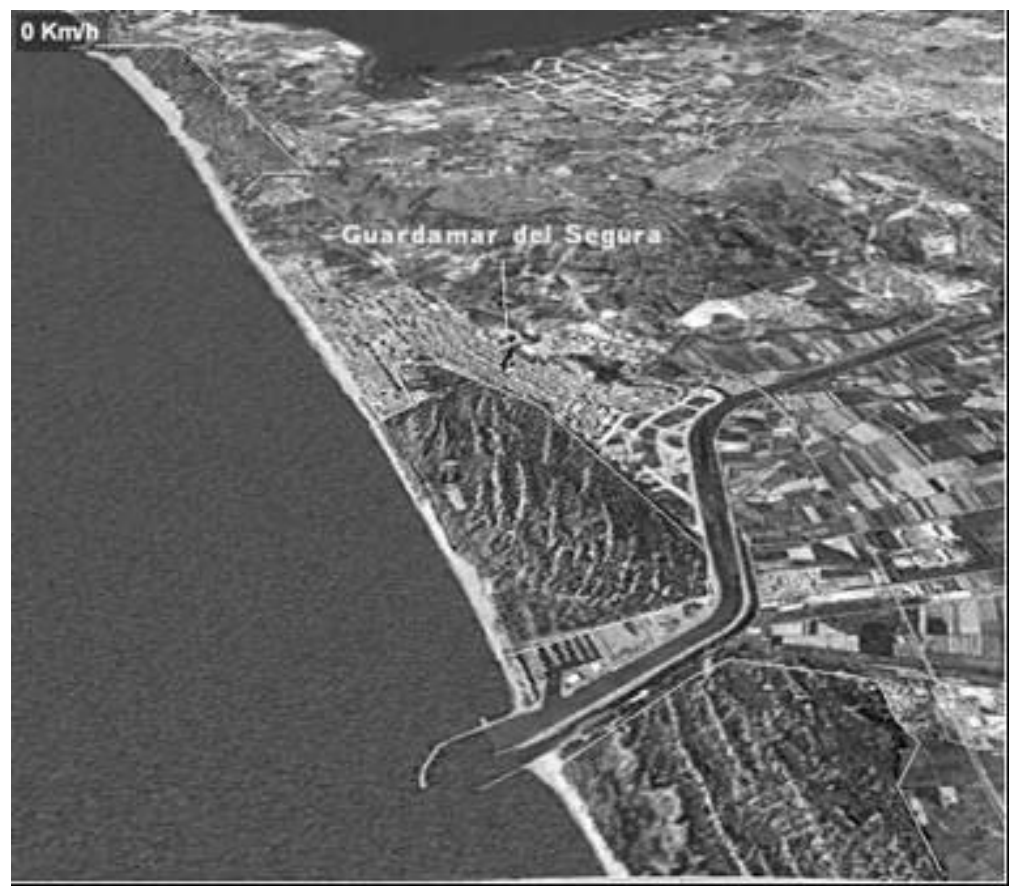

Figura 1. Fotografía aérea del Pinar de Guardamar de la Segura, (España).

\subsection{EQUIPO UTILIZADO}

Para estudiar el sistema radicular de las dos especies arbóreas se utilizó el RAMAC/ GPR fabricado por MALA GeoScience (Fig. 2), combinando tres tipos de antenas monoestáticas Shielded de100 MHz, $500 \mathrm{MHz}$. y $1000 \mathrm{MHz}$. Este equipo consta 
básicamente de un ordenador que permite hacer el setup de medida, una unidad de control que controla la emisión, recepción y registro de energía y una antena emisora-receptora de ondas electromagnéticas.

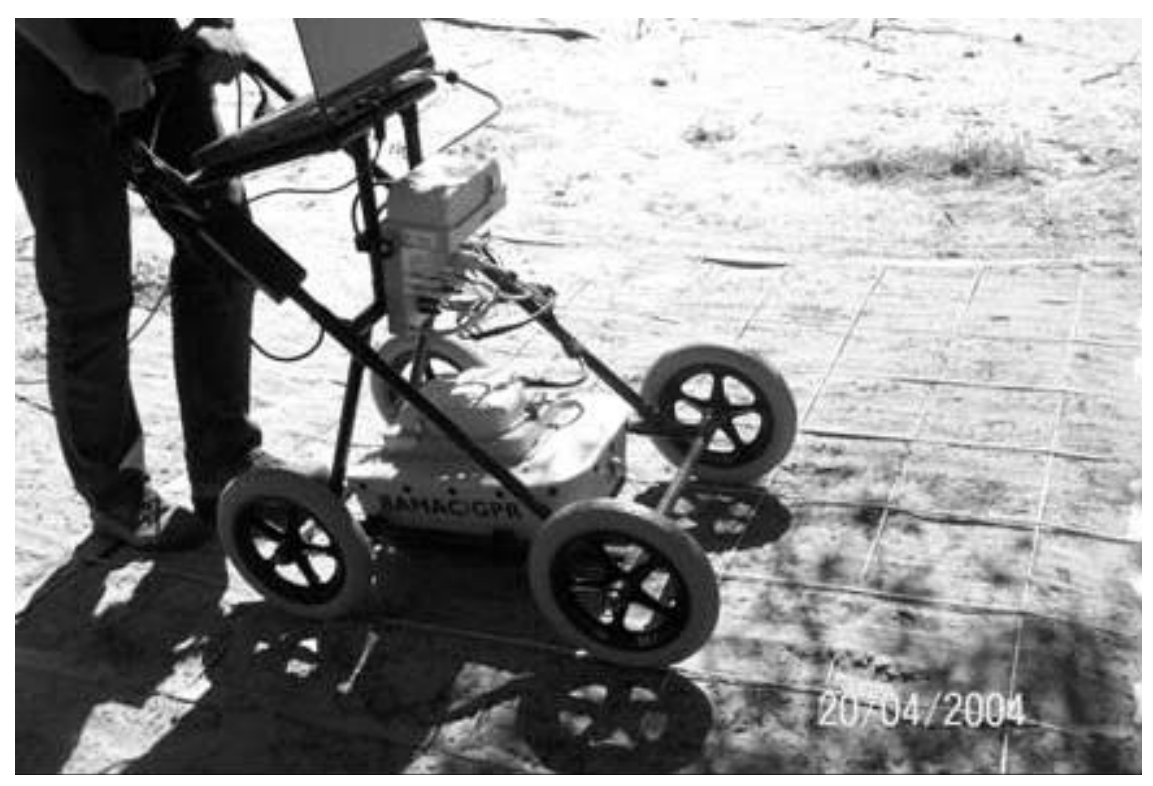

Figura 2. Equipo RAMAC/GPR utilizado para el estudio del sistema radicular.

\subsection{METODO}

La metodología empleada para el estudio del sistema radicular de las dos especies arbóreas dominantes consintió en:

1. Seleccionar árboles con un diámetro en la altura del pecho $(\mathrm{DBH})$ superior a 25 $\mathrm{cm}$. Luego se procedió a colocar a $1 \mathrm{~m}$ de la base del tronco una malla de $2 \mathrm{~m} \mathrm{x}$ $2 \mathrm{~m}$ con cuadricula de $25 \mathrm{~cm} \times 25 \mathrm{~cm}$ como se muestra en la figura 3, también se hizo un cuadrado de $4 \mathrm{~m} \times 4 \mathrm{~m}$ cuyo centro fuese el árbol y se trazaron perfiles cada $25 \mathrm{~cm}$ en los dos sentidos. Asimismo se utilizo una lámina de cartón de $1 \mathrm{~m}$ x $1 \mathrm{~m}$ marcando la separación de $15 \mathrm{~cm}$ y sentido de los perfiles como se observa en la figura 4. 

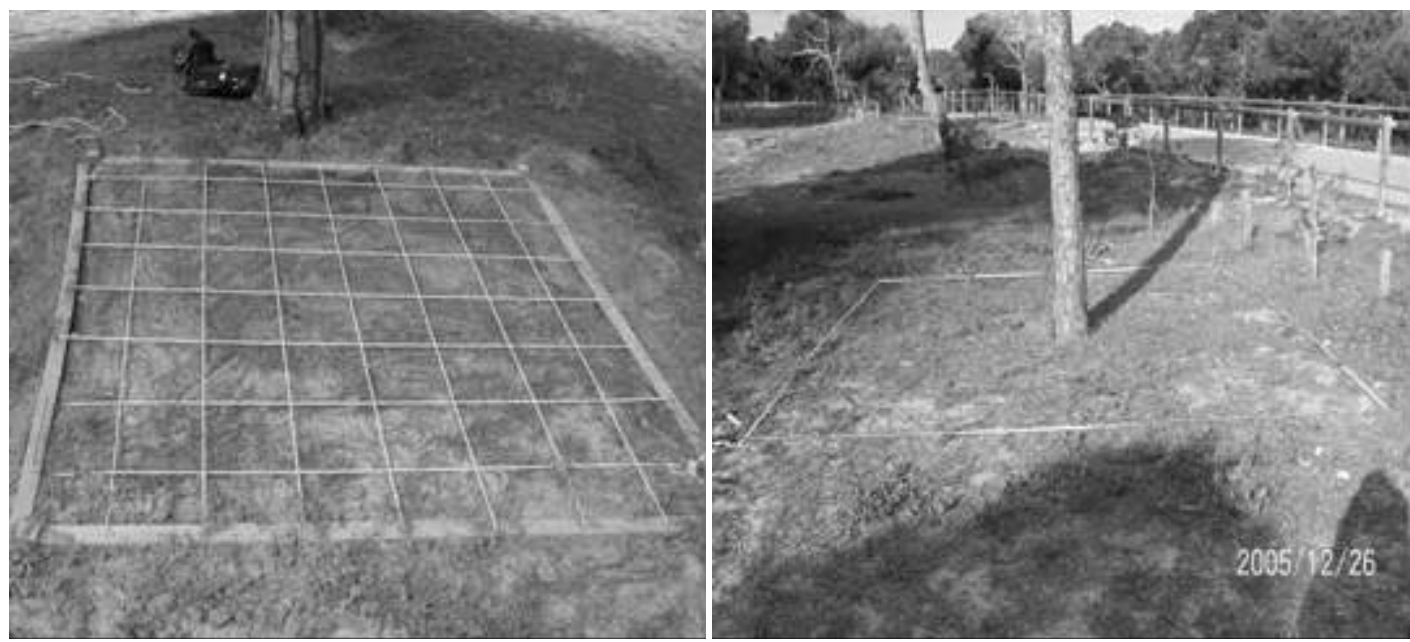

Figura 3. Preparación de las mallas que sirven de referencia al desenterrar las raíces y al mismo tiempo de guía para la antena de GPR.

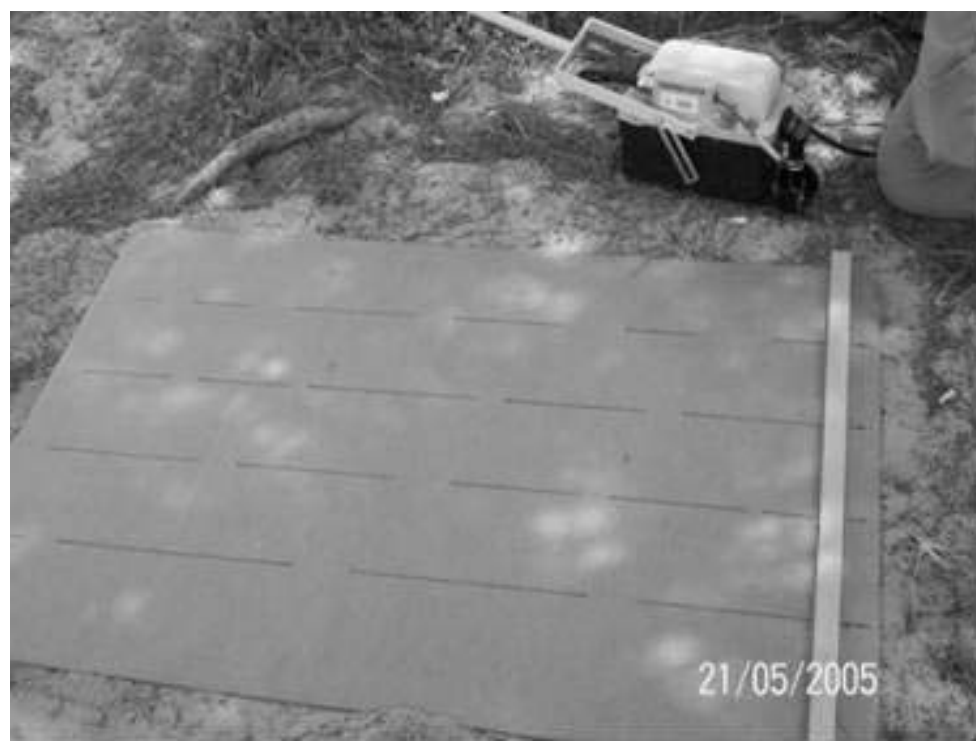

Figura 4. Lámina de cartón marcada con los perfiles y antena de $1000 \mathrm{MHz}$ usada para tomar registros.

2. Para la adquisición de datos se emplearon tres tipos de antenas: en primer lugar se usó una de $100 \mathrm{MHz}$ con una frecuencia de muestreo de $2000 \mathrm{MHz}$ y realizando perfiles perpendiculares a la costa. Seguidamente se utilizó una de $500 \mathrm{MHz}$ usando 
una frecuencia de $18000 \mathrm{MHz}$ y por último se utilizó una antena de $1000 \mathrm{MHz}$ con una frecuencia de muestreo de $29000 \mathrm{MHz}$.

3. Remoción de suelo: se hizo tanto en forma manual como mecánica. La primera se usó para tratar de ablandar el suelo (Fig.5a), posteriormente se utilizó una sopladora como se muestras en la figura $5 b$, esto con el propósito de retirar toda la arena posible pero sin alterar la posición de las raíces.

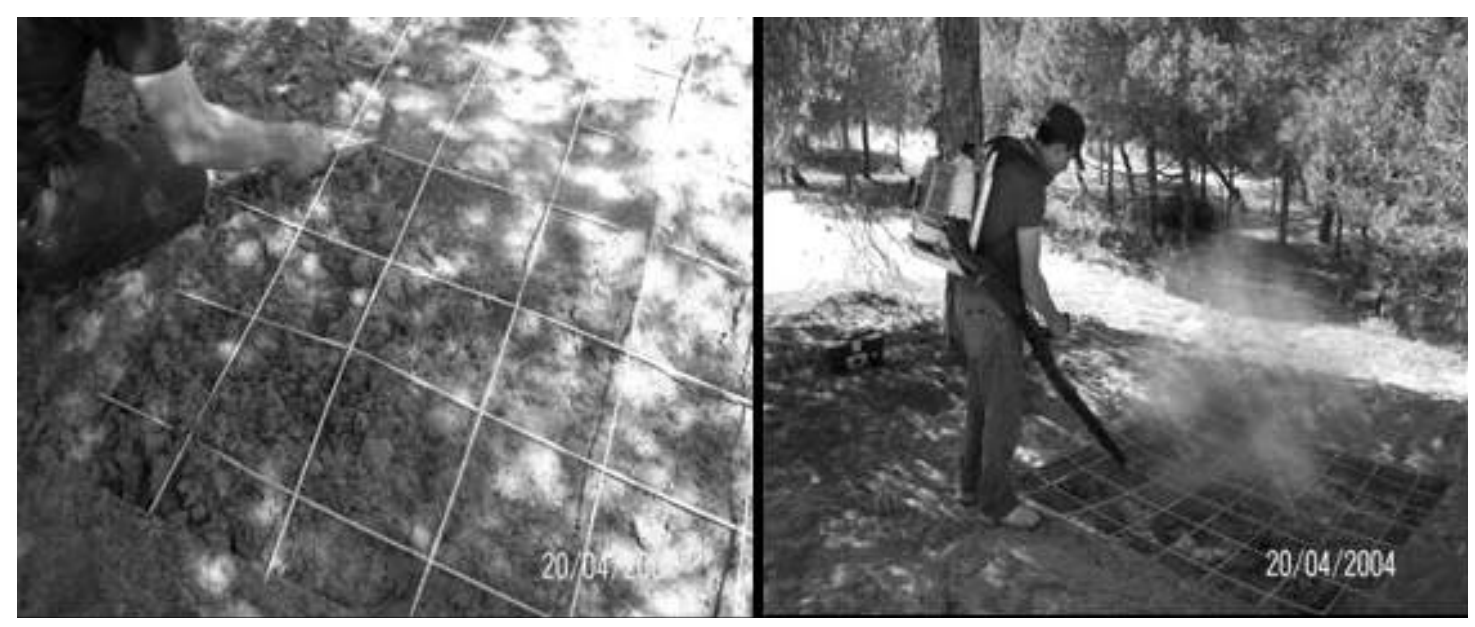

Figura 5a. Remoción manual del suelo.

Figura 5b. Remoción de suelo con sopladora.

4. Por último se contrastaron los datos adquiridos en el GPR con los observados, para ello se desenterraron las raíces en toda el área demarcada, la profundidad de excavación se hizo hasta aproximadamente $70 \mathrm{~cm}$. Una vez descubiertas las raíces se procedió a marcar cada una de estas, luego se tomo su profundidad e intercepción en cada eje y al final se midió su diámetro (Fig. 6).

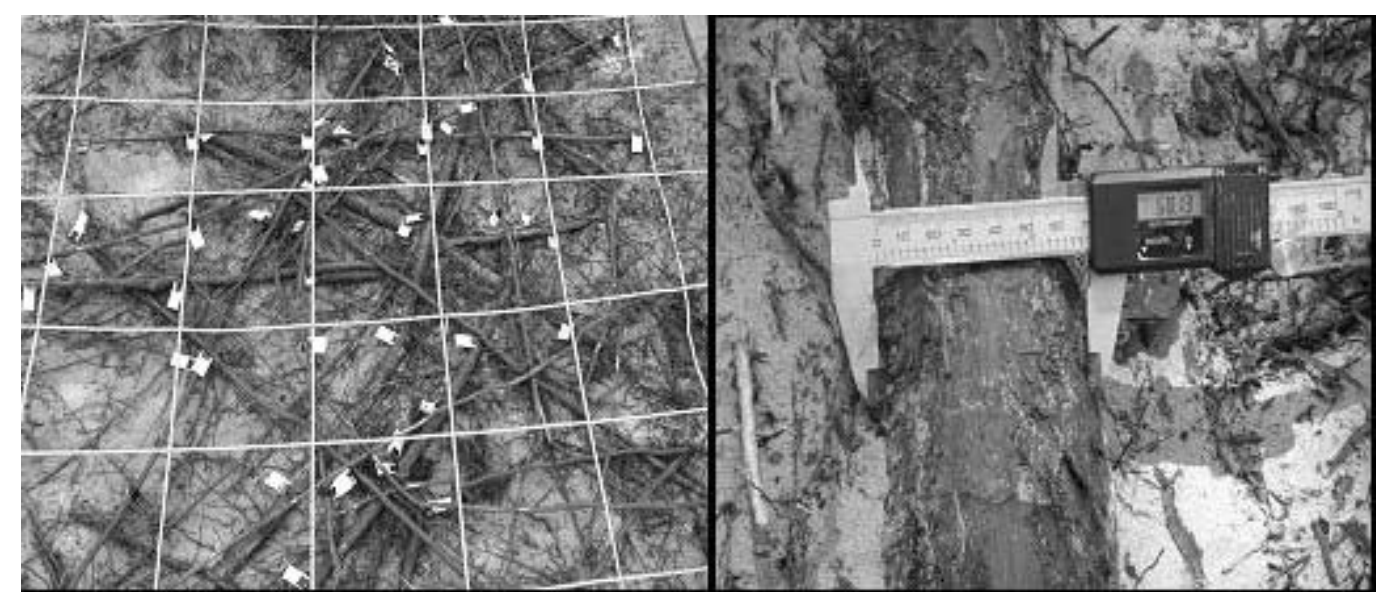

Figura 6. Identificación y medición de diámetro en las raíces desenterradas. 


\section{RESULTADOS Y DISCUSIÓN.}

El análisis de resultados empieza desde la organización de la campaña, siendo importante cada paso que se sigue, pues de ello depende la obtención de las conclusiones finales. Los registros algunas veces pueden interpretarse directamente. Otras veces se requiere de tratamiento especial de señales para mejorar la relación señal/ruido o para resaltar alguna anomalía específica, en este caso las producidas por las raíces. Por ello cuando nos era difícil observar las anomalías acudíamos a dos tipos de software, uno llamado Gradix INTERPEX LTD., E.E.U.U 1996 y otro software llamado Ramac GroundVision versión 1.4.1. Este último diseñado por Mala GeoScience, el cual incluye la adquisición y tratamiento de los datos.

El cálculo de la velocidad se realizó empleando la siguiente ecuación:

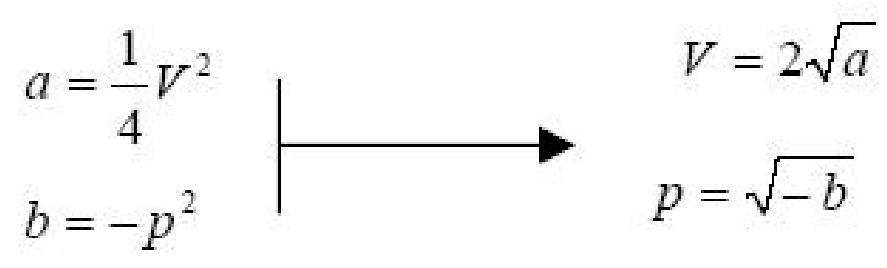

Donde:

$\mathrm{V}=$ velocidad de propagación

$\mathrm{P}=$ profundidad del objeto

a y b son parámetros conocidos por cálculo de la recta de ajuste, $a=89.88$ y b=0.2010

La velocidad calculada para las dunas de Guardamar del Segura fue de $90 \mathrm{~m} / \mathrm{ms}$. Esta velocidad permitió realizar la transformación de los datos de tiempo a profundidad y definir la profundidad real de las raíces.

En la figura 7 se muestra los resultados obtenidos de las 5 raíces enterradas con la antena monoestática de $500 \mathrm{MHz}$ utilizando una frecuencia de muestreo de $18000 \mathrm{MHz}$. En este radargrama se observa claramente la forma de las hipérbolas resultantes de cada una de las raíces enterradas durante la fase experimental. El eje $X$ representa la distancia $(m)$ total del perfil, mientras el eje $Y$ enseña la profundidad (m). 


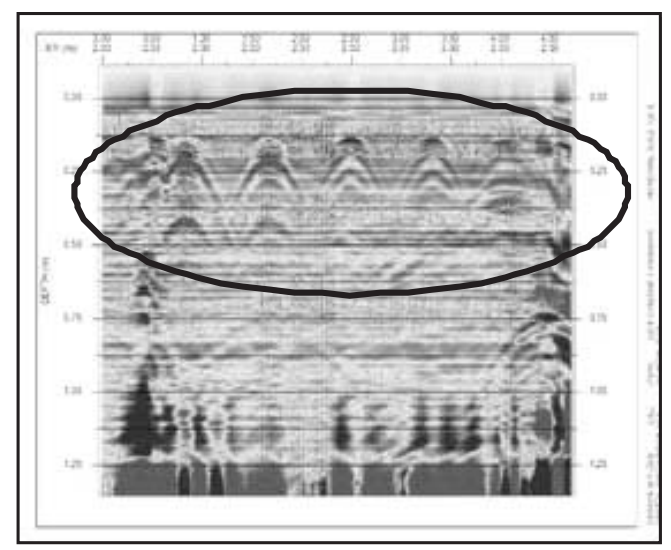

Figura 7. Perfil de calibración del GPR en las dunas de Guardamar, en este se observa las 5 raíces enterradas.

El tratamiento de este registro se hizo con el software Gradix Interpex, aprovechando la buena cantidad de filtros que tiene el programa y los cuales nos permiten eliminar o reducir aquellas señales que no correspondían a las anomalías producidas por la las raíces.

En la figura 8 se aprecian claramente las anomalías producidas por las raíces, en un de los perfiles perpendiculares a la costa medidos con esta misma antena (500 MHz), la longitud de estos fue aproximadamente de $26 \mathrm{~m}$. El tratamiento de los registros se hizo con el software Ramac groudvision, aplicando los filtros DC-Removal, Band Pass y Running Average. En estos se observa que la mayor densidad de raíces se localiza a profundidades comprendidas entre 0,20 y $0,80 \mathrm{~m}$, la profundidad alcanzada con esta antena y a una frecuencia de muestreo entorno a los $18000 \mathrm{MHz}$ fue de $2 \mathrm{~m}$. Además las excavaciones realizadas en más de 8 árboles permitieron corroborar que las raíces alcanzan una profundidad máxima de 0,80 m (figura 8) y en registros numéricos obtenidos en campo, generada en uno de estos desenterramientos.

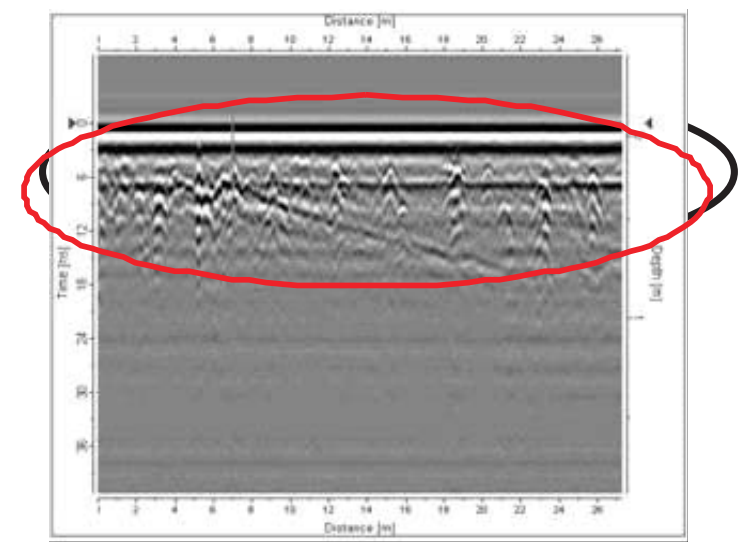

Figura 8. Perfiles de $26 \mathrm{~m}$ efectuados con antena apantallada de $500 \mathrm{MHz}$ donde se destacan las reflexiones relacionadas con las raíces. 
La figura 9 recoge un par de radargramas representativos de los registros obtenidos con la antena de $1000 \mathrm{MHz}$. El registro tiene una longitud de $90 \mathrm{~cm}$, la profundidad alcanzada con una frecuencia de $28000 \mathrm{MHz}$ fue de $50 \mathrm{~cm}$, los filtros utilizados fueron DC-Removal y Band pass. Usando esta antena se ha mejorado la resolución vertical del registro facilitando la identificación de reflectores producidos por las raíces, permitiendo identificar raíces de $1 \mathrm{~cm}$.

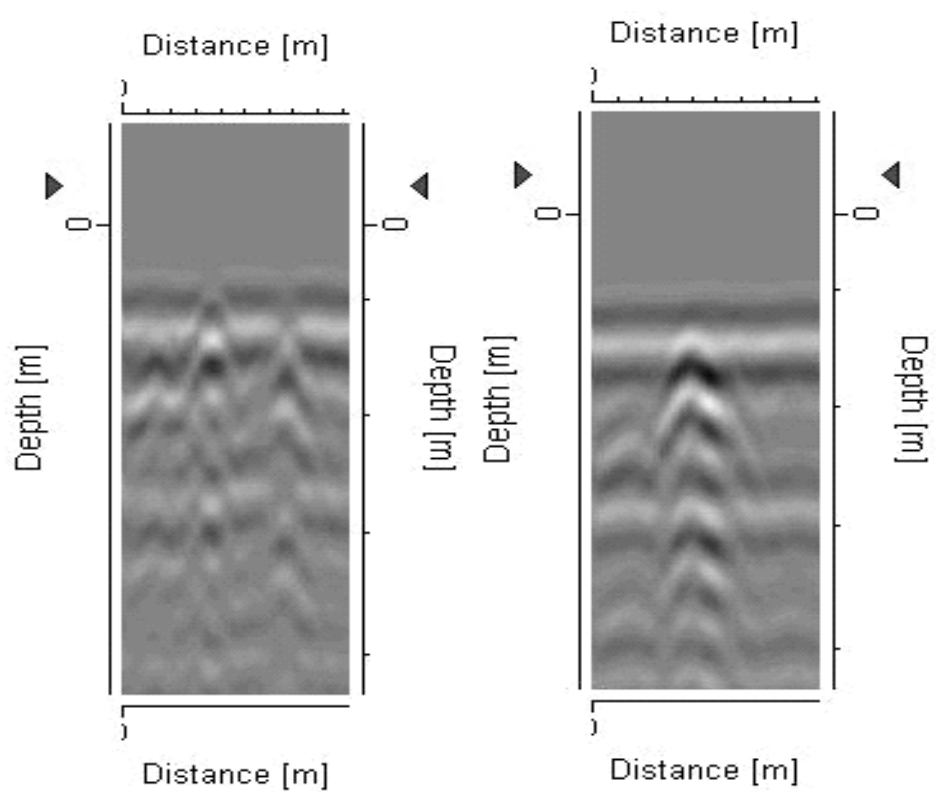

Figura 9. Radargramas obtenidos en dos perfiles registrados con la antena de $1000 \mathrm{MHz}$.

Con respecto a los datos obtenidos con la antena de $100 \mathrm{MHz}$ (Fig. 10) se pueden destacar los siguientes aspectos: al ser una antena de menor frecuencia su penetración en el subsuelo es mayor, permitiendo llegar a una profundidad de $10 \mathrm{~m}$ en un perfil de $76 \mathrm{~m}$. Si bien la amplitud de las reflexiones asociadas se ve atenuada con respecto a los registros anteriores, su menor resolución hace que sea un poco difícil apreciar algunas reflexiones internas relacionadas con las raíces. Sin embargo esta antena muestra con claridad otro tipo de anomalía (encerrada por un óvalo en la figura 10) en este caso sería el contacto arena-roca a partir de los 26 metros hasta los 76 metros (escala horizontal superior de la figura 10) y a profundidades comprendidas entre 0,10 y 1,50 m (escala vertical derecha) como se muestra en este radargrama. Es posible que estas reflexiones estén relacionadas con la estratigrafía interna de las dunas. 


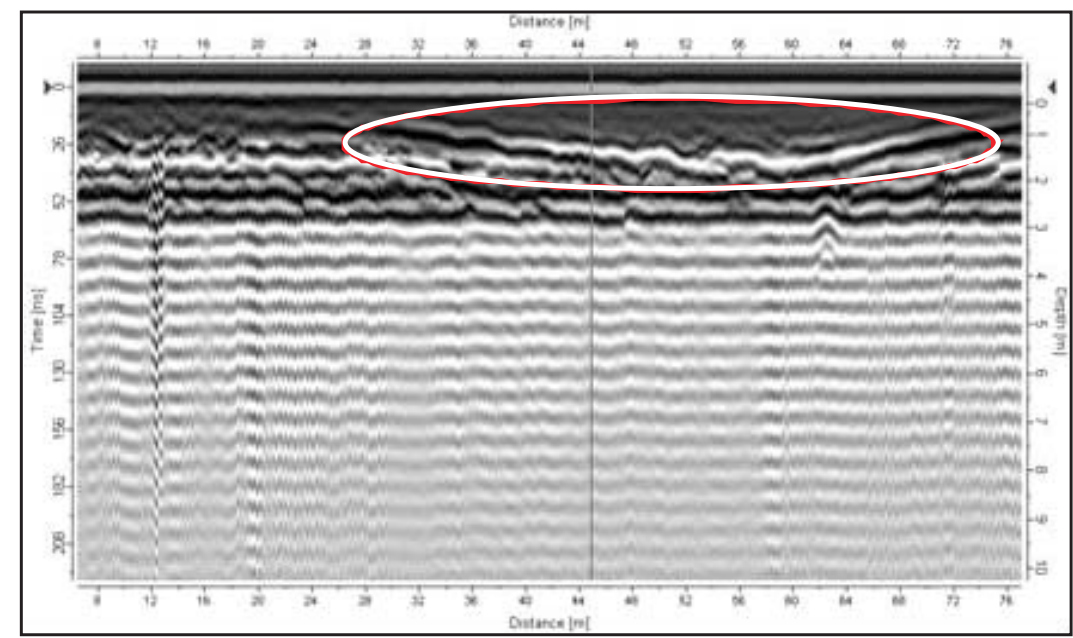

Figura 10. Perfil de $76 \mathrm{~m}$ efectuado con una antena apantallada de $100 \mathrm{MHz}$ donde se detectan reflexiones ocasionadas por otro tipo de anomalías.

Cuando las condiciones del suelo son favorables, es decir para suelos bien drenados, homogéneos y con poca salinidad, el georradar es un equipo muy eficiente para medir la biomasa subterránea. Butnor et al., [4] lo ha empleado en suelos forestales (con buen contenidos de materia orgánica), logrando detectar raíces de hasta de $5 \mathrm{~mm}$ usando una antena de $1.5 \mathrm{GHz}$, sin embargo en este estudio se han obtenido buenos resultados con antena de $500 \mathrm{MHz}$, logrado detectar raíces del orden de $10 \mathrm{~mm}$ en suelos arenosos y expuestos a espray marino.

\section{CONCLUSIONES}

Después de haber realizado varios ensayos con diferentes antenas $(100 \mathrm{MHz}, 500 \mathrm{MHz}$ y $1000 \mathrm{MHz}$ ) y múltiples frecuencias de muestreo, estamos convencidos que la técnica del GPR es una excelente alternativa para el estudio del sistema radicular de especies arbóreas en ecosistemas dunares.

El método del GPR es una técnica geofísica que permite estudiar anomalías, en nuestro caso las producidas por las raíces sin generar ningún tipo de impacto ambiental, convirtiéndose así en un método no invasivo y no agresivo.

La antena que mejores resultados proporciono en este tipo de ambientes fue la de 500 $\mathrm{MHz}$, utilizando una frecuencia de muestreo de $18000 \mathrm{MHz}$. Esta nos permitió obtener una mayor resolución de las anomalías producidas por las raíces. 
La profundidad de investigación alcanzada con la antena de $500 \mathrm{MHz}$ fue de 3,5 m, profundidad suficiente para estudiar el sistema radicular de las especies de pino del ecosistema dunar de Guardamar del Segura.

La antena de $1000 \mathrm{MHz}$ ofrece buenos resultados pero solo a profundidades no mayores de $50 \mathrm{~cm}$.

Los resultados observados con la antena de $100 \mathrm{MHz}$ muestran que esta alcanza una mayor profundidad de investigación, pero se obtiene menor resolución de las anomalías producidas por las raíces.

\section{BIBLIOGRAFÍA}

[1] ALDEGUER, M. 1989. Dinámica litoral y génesis dunar. En: Escarré A, Martín, J y Seva, E. (eds.) Estudios sobre el medio y la biocenosis en los arenales costeros de la Provincia de Alicante, cap III, pp. 27-42. Diputación Provincial de Alicante.

[2] ANNAN, A.P. y J.P. DAVIS, 1976. Impulse Radar Soundig in Permafrost. Radio Science, 11 (4): 383-394.

[3] BARBOUR, M.G., DE JONG, T.M. y PAVLIK, B.M. 1985. Marine beach and dune plant communities. En: Chabot, B.F. y Mooney, H.A. (eds.) Physiological ecology of North American plant communities. Chapman y Hall, New York., 296-322.

[4] BUTNOR, J. R., DOOLITTLE, J. A., KRESS, L., COHEN, S. y JOHNSEN, K. H. 2001. Use of ground-penetrating radar to study tree roots in the southeastern United States. journal Tree Physiology 21, 1269-1278.

[5] CARCIONE, J.M., 1996. Ground radar simulation for archaeological application. Geophysical Prospecting, 44: 871-888.

[6] GLOPPER, R.J. 1964. About the water contenent and shrinkage of some Dutch lacustrine and marine sedements. Neth. J. Agric, 12: 221-226.

[7] GLOVER J.M., 1987. The use of sub-surface radar for shallow site investigations. Ph. D. Thesis, Kings College, University of London.

[8] GOODMAN, D. 1994. Ground-penetrating radar simulation in engineering and archaeology. Geophysics, 59: 224-232. 
[9] HRUSKA, J. CERMÁK, J y SVATOPLUK SUSTEK. 1999. Mapping tree root systems with ground-penetrating radar. Journal Tree Physiology 19, 125-130.

[10] MARTíN, J., SEVA, A. y ESCARRÉ, A. 1989. Características del sustrato dunar. En: Escarré, A., Martín, J y Seva, E. (eds.) Estudios sobre el medio dunar y la biocenosis.

[11] NORDSTROM, K. 1994. Beaches and Dunes of human-altered coasts; Progress in physical Geography, 18 (4): 497-516.

[12] OOSTING, J.H. 1954. Ecological processes an vegetation of maritime stand in the Southeastern United States. Botanical Review, 20: 226-262.

[13] PASKOFF, R. 1993. Côtes en danger ; Masson, Paris, 250 pp.

[14] PÉREZ GRACIA, J.A., CANAS, LL.G., PUJADES, J.C., CASELLES, O., RANWELL, D. 1972. Ecology of salt marshes and salt dunes. Chapman and Hall. Londres. pp. 135-200.

[15] RANWELL, D. 1972. Ecology of salt marshes and salt dunes. Chapman and Hall. Londres, pp. 135-200.

[16] RUBIN, L.A. y FOWLER J.C. 1977. Ground probing radar for delineation of rock features. Engineering Geology, 12: 163-170.

[17] SALISBURY, E.J. 1952. Downs and dunes, their Plant Life and its Environment Bell, London.

[18] ULRIKSEN, C.P., 1982. Application of impulse radar to civil engineering. Ph. D. Thesis, Lund University of Technology.

[19] WILLIS, A.J., FOLKES, B.F., HOPE-SIMPSON, J.F. y YEMM, E.W. 1959. Braunton Burrows: the dune system and its vegetation. Part II. Journal of Ecology, 47: 249-288. 